\title{
Article \\ FAST AND ACCURATE BACKGROUND RECONSTRUCTION USING BACKGROUND BOOTSTRAPPING
}

\author{
Bruno Sauvalle ${ }^{1 *}$, Arnaud de La Fortelle ${ }^{1}$ \\ 1 Centre de Robotique, Mines ParisTech PSL University, 75006 Paris, France \\ * Correspondence: bruno.sauvalle@mines-paristech.fr
}

\begin{abstract}
The goal of background reconstruction is to recover the background image of a scene from a sequence of frames showing this scene cluttered by various moving objects. This task is fundamental in image analysis, and is generally the first step before more advanced processing, but difficult because there is no formal definition of what should be considered as background or foreground and the results may be severely impacted by various challenges such as illumination changes, intermittent object motions, highly cluttered scenes, etc. We propose in this paper a new iterative algorithm for background reconstruction, where the current estimate of the background is used to guess which image pixels are background pixels and a new background estimation is performed using those pixels only. We then show that the proposed algorithm, which uses stochastic gradient descent for improved regularization, is more accurate than the state of the art on the challenging SBMnet dataset, especially for short videos with low frame rates, and is also fast, reaching an average of $52 \mathrm{fps}$ on this dataset when parameterized for maximal accuracy using GPU acceleration and a Python implementation.
\end{abstract}

Keywords: background reconstruction; background initialization; background generation; motion detection; background subtraction; scene parsing

\section{Introduction}

We consider in this paper the task of static background reconstruction: starting from a sequence of images $X_{1}, . ., X_{N}$ of a scene showing moving objects, for example cars, bikes or pedestrians, the goal is to recover the image of the background of this scene, without any of the moving objects. This task is fundamental in image analysis: The moving objects appearing in the scene may be considered as a nuisance, and background reconstruction allows to remove them completely and focus on the analysis of the background, for example to localize or map the scene. More frequently, for example for video surveillance or traffic monitoring, the moving objects are the main object of interest and the background itself is considered as a nuisance, so that background reconstruction is a first step which can be used to extract and analyse the moving objects of the scene. The task of background reconstruction should not be confused with the task of background modeling which involves building a statistical model of the background image whereas the task of background reconsctruction requires to predict a unique background image.

It is often assumed that all the images $X_{1}, . ., X_{N}$ share the same background, which is then called a static background. In this case, the output of the algorithm is composed of only one background image $\hat{X}$. It is however also possible that the backgrounds are slightly different in each image, for example if the illumination conditions change or if the camera is moving. In this situation, we expect a background reconstruction algorithm to output a sequence of backgrounds $\hat{X}_{1}, . ., \hat{X}_{N}$ and we say that the background reconstruction is dynamic. In this paper, we consider the problem of static background reconstruction. 
This problem is a difficult because there is no formal definition of what should be considered as background or foreground. Moving trees, fountains, moving shadows are examples of instances which are usually considered as belonging to the background although they show moving features. Other challenges like illumination changes or the presence of objects staying still for a short time (a problem called intermittent motion) may severely impact the quality of a backgroud reconstruction model.

The paper is organized as follows : In section 2, we review related work in static background reconstruction. In section 3 we describe the proposed algorithm. Experimental results are then provided in section 4 .

\section{Related work}

One should distinguish between online methods, where the length of the dataset is unknown and the background reconstruction algorithm has to update the background model in real-time and batch methods, where the algorithm is provided with a fixed dataset. The method proposed in this paper is a batch method.

The current state of the art models for unsupervised fixed background reconstruction are the Superpixel motion detection algorithm (SPMD) [1] and LabGen-OF [2]

SPMD first selects the longest sequence with stable illumination, then uses superpixel segmentation, and removes all superpixels with contain at least one moving pixel. The various pixel values associated to one pixel position are then clustered, and the median value of the best cluster is selected to produce the background value.

LabGen [3] assumes that a background/foreground segmentation algorithm is available. For a given spatial patch, it selects the frames where the spatial patch has the lowest number of foreground pixels, and then performs a pixel-wise median filtering on theses patches. LabGen-OF is a variant of this algorithm which uses an optical flow algorithm [2]. LabGen-semantic is another variant with uses a supervised semantic segmentation model [4].

Temporal median filtering (TMF) is a very simple algorithm which computes the background color for a pixel $p$ as the median of the colors of this pixel on all the images $X_{1}, \ldots, X_{N}$. Despite its simplicity, this algorithm and its variant TMFG using gaussian filtering [5] performs very well on several scene categories.

The FSBE algorithm (frame selection and background estimation) [6] assumes that an optical flow algorithm is available. It first selects a sequence of frames where the illumination conditions do not change too much. Using the optical flow algorithm, it classifies as background all pixels which have an optical flow magnitude below some threshold and corrects this classification if it detects high dynamic motion or foreground intermittent motion in the sequence. It then takes the pixel-wise average of the selected background pixels.

Photomontage [7] builds the background as a seamless montage composed of patches extracted from the images $X_{1}, . ., X_{N}$ so that the likelihood of the color at each pixel is maximum with respect to the probability distribution function formed from the color histogram of all pixels in the span.

The BEWIS [8] and SOBS algorithm [9-11] involve weightless neural networks, which are used as containers to build a statistical model of the background.

The current top performing algorithms for background reconstruction do not use deep learning techniques, but several papers have proposed to use them for fixed background reconstruction :

FC-Flownet [12] is a convolutional network with an architecture similar to a U-net which is used to predict a background from a set of 20 color images in a single inference step. Due to memory restrictions, the images are cut in superposed $64 \times 64$ patches, and the 20 patches associated to one location are given as input to the convolutional network. The ouput patches are then aggregated to build the background. The network is trained end-to-end using samples and ground truths coming from 54 different sequences. 
BM-Unet [13] is a background reconstruction model which also uses a U-net network but is trained without any supervision or ground-truth data and can perform both fixed and dynamic background reconstruction. For fixed background reconstruction, it is trained with pairs of random images sampled from one frame sequence. Using the first image, the U-net network predicts a probability distribution over the possible 255 values of each pixel of the output image, and the second image is used as a target.

It is possible to use a dynamic background reconstruction model to perform fixed background reconstruction by simply selecting one of the reconstructed backgrounds $\hat{X}_{1}, . ., \hat{X}_{n}$ to be the final background reconstruction $\hat{X}$. The Motion-assisted spatiotemporal clustering of low-rank algorithm (MSCL) [14], which is a dynamic background reconstruction model using robust principal component analysis (RPCA), is able to get better results than state of the art fixed background reconstruction models on the SBMnet dataset using this method. It should however be noted that this approach is not directly comparable to those models because it requires some human supervision to select the final frame $\hat{X}$ from $\hat{X}_{1}, \ldots, \hat{X}_{n}$.

We refer to the surveys $[15,16]$ for a more detailed description of related work.

\section{Proposed algorithm for background reconstruction}

\subsection{Motivation}

We have noted in the review of previous work the good results of temporal median filtering, despite its simplicity, and observe that the two best unsupervised algorithms for background reconstruction, SPMD and LabGen-OF, also use some form of temporal median filtering. One can intuitively understand that background reconstruction involves performing some form of averaging of the input frames, and that computing the median will give better results than computing the average of the frames because the median is more robust to outliers.

We note however that using median filtering on color images may lead to inconsistencies. Let's for example consider RGB images showing a red background with large green and blue foreground objects. Assume that in the sequence considered, each red background pixel is masked by a green object during $26 \%$ of sequence duration and by a blue object during another $26 \%$ of the sequence duration. The red color channel of any pixel will then be equal to zero during $52 \%$ of the sequence, and the blue and green channels are also equal to zero during $74 \%$ of the sequence. As a consequence, the result of median filtering on such a sequence is a uniform black image, which is clearly not satisfactory.

One can think that a better method to select the background color of an image from a frame sequence would be first to guess in each frame which pixels are background pixels and then to consider only those pixels for temporal median filtering. However, to be able to guess which pixels are background pixels, we need to have some estimate of the background. The main idea introduced in this paper is that we can successfully build an iterative optimization process for background reconstruction, using the current estimate of the background to guess which pixels are background pixels and then refining the estimate of the background by performing temporal median filtering on those pixels only.

\subsection{Bootstrap weights}

We observe that temporal median filtering can be described as a minimization problem associated to a $L_{1}$ error loss. More precisely, for a sequence of color images $X_{1}, \ldots, X_{N}$ of size $h \times w$, noting $x_{n, c, i, j}$ the value (normalized in the range $\left.[0,1]\right)$ of the pixel associated to the image $X_{n}$ and the color channel $c$ at position $(i, j)$ with $1 \leq i \leq h$ and $1 \leq j \leq w$, the $L_{1}$ loss function can be described as

$$
\mathcal{L}_{1}\left(\hat{X},\left(X_{n}\right)_{1 \leq n \leq N}\right)=\frac{1}{N} \sum_{n=1}^{N} L_{1}\left(\hat{X}, X_{n}\right)
$$


with

$$
L_{1}\left(\hat{X}, X_{n}\right)=\frac{1}{h w} \sum_{i=1, j=1}^{h, w} \sum_{c=1}^{3}\left|\hat{x}_{c, i, j}-x_{n, c, i, j}\right|,
$$

and it is immediate that if we take each $\hat{x}_{c, i, j}$ to be a median of the sequence $\left(x_{n, c, i, j}\right)_{1 \leq n \leq N}$, then we get a minimum of this loss function, considering that the gradient of $\mid \hat{x}_{c, i, j}-$ $x_{n, c, i, j} \mid$ with respect to $\hat{x}_{c, i, j}$ is equal to 1 if $\hat{x}_{c, i, j}-x_{n, c, i, j}>0$ and -1 if $\hat{x}_{c, i, j}-x_{n, c, i, j}<0$. We bootstrap the current estimate of the background to build a soft foreground / background segmentation mask, and smoothly restrict this loss function to the background pixels only : Let's note $l_{n, i, j}$ the sum of the $L_{1}$ errors for each color at the pixel $(i, j)$ between the predicted image $\hat{X}$ and the input image $X_{n}$ for all the color channels :

$$
l_{n, i, j}=\sum_{c=1}^{3}\left|\hat{x}_{c, i, j}-x_{n, c, i, j}\right|
$$

If at least one of the color channels give a high error, then $l_{n, i, j}$ is large and we will consider that the pixel $(i, j)$ of the image $X_{n}$ is a foreground pixel. We then build a soft foreground mask $m_{n} \in[0,1]^{h \times w}$ for the image $X_{n}$ using the formula

$$
m_{n, i, j}=\tanh \left(\frac{l_{n, i, j}}{\tau_{1}}\right)
$$

Where $\tau_{1}$ is some positive hyperparameter, which can be considered as a soft threshold. This mask will however be noisy (cf Figure 1). We then compute a spatially smoothed version $\tilde{m}_{n, i, j}$ of this mask by averaging using a square kernel of size $(2 k+1) \times(2 k+1)$, with $k=\lfloor w / r\rfloor$ (where $w$ is the image width and $r$ is some integer hyperparameter):

$$
\tilde{m}_{n, i, j}\left(\hat{X}, X_{n}\right)=\frac{1}{(2 k+1)^{2}} \sum_{l=-k, p=-k}^{l=k, p=k} m_{n, i+l, j+p}
$$

The associated pixel-wise weight $w_{n, i, j}^{\text {bootstrap }}$ is then defined as :

$$
w_{n, i, j}^{\text {bootstrap }}=e^{-\beta \tilde{m}_{n, i, j}}
$$

where $\beta$ is some positive hyperparameter,which we call the bootstrap coefficient.

\subsection{Optical flow weights}

We have seen that background reconstruction algorithms could be improved by using informations provided by optical flow models to remove parts of an image showing moving objects. We use the same approach to improve the loss functions $\mathcal{L}_{1}$ :

We use an external algorithm (OpenCV implementation of Dense Inverse Search algorithm [17]) to get an estimate of the magnitude $\phi_{n, i, j}$ of the optical flow associated to each pixel $(i, j)$ of an image $X_{n}$. We chose this algorithm because it is very fast compared to other available optical flow implementations. We first normalize $\phi_{n, i, j}$ with respect to the image width $w$ and then define an optical flow mask $\mu_{n, i, j}$ using the formula

$$
\mu_{n, i, j}=\min \left(1, \frac{\phi_{n, i, j}}{w \tau_{2}}\right) \text {, }
$$

where the hyperparameter $\tau_{2}$ can also be considered as a threshold. The weight associated to this optical flow mask is defined as :

$$
w_{n, i, j}^{\mathrm{OF}}=e^{-\phi \mu_{n, i, j}}
$$




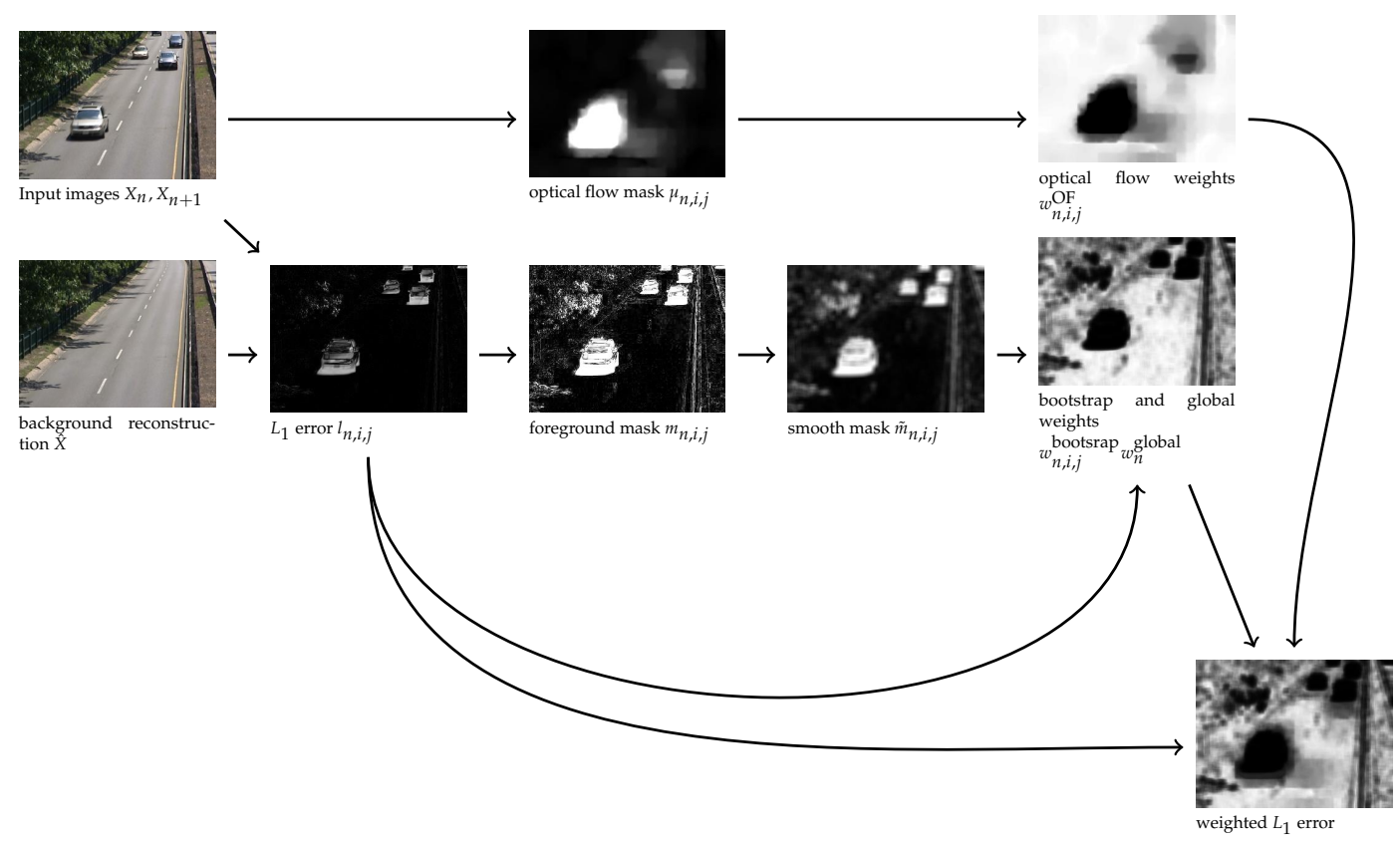

Figure 1. schematic of loss function computation (Images are normalized in the range $[0,1]$ )

where $\phi$ is another positive hyperparameter, considering that a pixel is not likely to be a background pixel if the associated optical flow magnitude is large. $\phi$ is however set to zero for short videos (less than ten images), considering that optical flows computed from sequences with very low frame rates are not reliable.

\subsection{Abnormal image weights}

If the number of images in the dataset is large, we can afford to give a low weight to images which appear to be abnormal, for example if the illumination conditions are different on these images compared to the predicted background, or if there are too many pixel errors on the image. We then first compute the average $L_{1}$ error $\bar{l}_{n}$ of the image $X_{n}$ as :

$$
\bar{l}_{n}=\frac{1}{h w} \sum_{i, j} l_{n, i, j}
$$

and define a global weight associated to each image $X_{n}$ as

$$
w_{n}^{\text {global }}=e^{-\gamma \bar{l}_{n}},
$$

where $\gamma$ is another positive hyperparameter. We use this global weight if the size of the dataset is greater than 10 .

\subsection{Management of intermittent motion}

Existing benchmarks for background reconstruction require that objects which remain still for a long time in the sequence be considered as foreground objects if they are moving during some part of the sequence. This challenge is very difficult and is not addressed by the previous weights. In order to handle it, we follow MSCL [14] and remove from the frames sequence all frames which are not showing any motion. More precisely, we first compute the maximum $\mu_{n}^{*}$ of the optical flow mask values $\mu_{n, i, j}$ of the image $X_{n}$ as defined in previous section, and remove this image if $\mu_{n}^{*}<\tau_{3}$, where $\tau_{3}$ is another threshold hyperparameter. The motivation of this suppression is that it appears that images containing still foreground objects are often motionless images, so that removing them improves the robustness of the proposed model against the intermittent motion challenge. We apply this motionless frame suppression when the 
number of frames in the sequence is higher than 10, considering as in previous section, that removing frames when the number of frames is very low will impact negatively the quality of the results. We note $N^{\prime} \leq N$ the number of frames after motionless frame suppression.

\subsection{Statement of the optimization problem}

Finally, the loss function is adapted using these weights and becomes the following:

$$
\mathcal{L}_{W}\left(\hat{X},\left(X_{n}\right)_{1 \leq n \leq N^{\prime}}\right)=\frac{1}{N^{\prime} h w} \sum_{n=1, i=1, j=1}^{N^{\prime}, h, w} w_{n}^{\text {global }} w_{n, i, j}^{\text {bootstrap }} w_{n, i, j}^{O F} \sum_{c=1}^{3}\left|\hat{x}_{c, i, j}-x_{n, c, i, j}\right|
$$

We are then interested to solve the following optimization problem : Considering the dataset $\left(X_{n}\right)_{1 \leq n \leq N^{\prime}}$, find an image $\hat{X}$ so that, when the weights $w_{n}^{\text {global }}$ and $w_{n, i, j}^{\text {bootstrap }}$ are considered as constants, the loss function $\mathcal{L}_{W}\left(\hat{X},\left(X_{n}\right)_{1 \leq n \leq N^{\prime}}\right)$ is minimal with respect to $\hat{X}$.

We can find a solution to this problem by performing an iterative computation of the weighted median of the images using the various weights defined in the previous paragraph followed by an update of the weights. We observe however that the images produced using this method are not smooth and that additional regularization is necessary. We then propose to use stochastic gradient descent on the loss function $\mathcal{L}_{W}\left(\hat{X},\left(X_{n}\right)_{1 \leq n \leq N^{\prime}}\right)$ using standard deep learning tools. The pixel values $\hat{x}_{c, i, j}$ are then considered as parameters and optimized using stochastic gradient descent.

It should be noted that performing a stochastic gradient descent on this loss function is not equivalent to minimizing it: During the optimization process, the weights $w_{n, i, j}^{\text {boostrap }}$ and $w_{n}^{\text {global }}$ depend on the current estimation of the background and change: We then call these weights dynamic weights. At each iteration they are however considered as fixed so that we do not compute and use the gradient of the loss function with respect to the value of these weights.

\section{Evaluation of the proposed model}

Two public benchmarks are available for the evaluation of fixed background reconstruction models : the SBMnet dataset [18] and the SBI dataset [19]. We first provide a quantitative evaluation of the proposed model on those two datasets. We then perform an ablation study and some computation speed measurements.

\subsection{Implementation details}

A desktop computer with an Intel Core i7 7700K@4,2GHz CPU and a Nvidia RTX 2080 TI GPU is used for this experiment.The model is implemented in Python using the Pytorch framework and is publicly available on the Github platform. We use the Adam optimizer, with learning rate 0.03 and batch size 64 , reduced by a factor of 10 when $3 / 4$ of the epochs have been computed. The number of epochs depends on the size of the dataset and is adjusted so that the total number of optimization iterations is close to 3000 , with a minimum of two epochs. In order to accelerate computations, each frame sequence is fully loaded in the GPU video RAM during the optimization process. A manual hyperparameter search has been performed using the video sequences of the SBI and SBM datasets for which a ground truth is available. The hyperparameters have then been set to the following values : $\beta=6, \phi=2, \gamma=3, r=75, \tau_{1}=0.25, \tau_{2}=$ $255 / 40000, \tau_{3}=240 / 255$. Before starting the optimization, background image pixel color values are initialized with random numbers sampled from a uniform distribution between 0 and 1. The DIS optical flow OpenCV implementation is used with the FAST preset mode. In order to get a low gradient when $l_{n, i, j}$ is close to zero, we replace the expression $\left|\hat{x}_{c, i, j}-x_{n, c, i, j}\right|$ with a smooth $L_{1}$ loss using a threshold equal to 3 (assuming the pixel values are scaled in the range 0 -255): When $\left|\hat{x}_{c, i, j}-x_{n, c, i, j}\right|$ is lower than 3 , we 
replace it with the quadratic expression $0.5\left(\hat{x}_{c, i, j}-x_{n, c, i, j}\right)^{2} / 3$, otherwise we replace it with $\left|\hat{x}_{c, i, j}-x_{n, c, i, j}\right|-0.5 \times 3$.

\subsection{Evaluation on SBMnet dataset}

The SBMnet dataset is composed of 79 sequences, which have been selected to cover a wide range of challenges and are representative of typical indoor and outdoor visual data captured today in surveillance, smart environment, and video database scenarios. The dataset includes the following eight categories with associated challenges: basic, intermittent motion, clutter, jitter, illumination changes, background motion, very long and very short. Although this dataset is freely available on the SBMnet website (www.SceneBackgroundModeling.net), ground truth images are publicly available for only 18 frame sequences, either on the SBMnet website or on the SBI dataset website. In order to benchmark a new algorithm, one has to submit the predicted fixed background images associated to each frame sequence to the website, which performs the evaluation of the submitted results.

Six criteria are computed to evaluate the accuracy of background reconstruction :

- $\quad$ Average Gray-level Error (AGE)

- Percentage of Error Pixels (pEPs)

- Percentage of Clustered Error Pixels (pEPs)

- Multi-Scale Structural Similarity Index (MS-SSIM)

- Peak-Signal-to-Noise-Ratio (PSNR)

- Color image Quality Measure (CQM)

We refer to [18] for the full definition of these criteria. A good background reconstruction should minimize the criteria AGE, $p E P s$ and pEPs, but maximize the criteria MS-MSSIM, PSNR and CQM. We have computed the 79 background images using the proposed algorithm and uploaded the reconstructed backgrounds to the SBMnet website, which provided the evaluation results and made them publicly available on the website.

Table 1. evaluation results per criteria on the SBMnet 2016 dataset. $\downarrow$ indicates lower score is better, $\uparrow$ indicates higher score is better.

\begin{tabular}{lrrrrrr}
\hline Method & $\begin{array}{r}\text { Average } \\
\text { AGE } \downarrow\end{array}$ & $\begin{array}{r}\text { Average } \\
\text { pEPs } \downarrow\end{array}$ & $\begin{array}{r}\text { Average } \\
\text { pCPES } \downarrow\end{array}$ & $\begin{array}{r}\text { Average } \\
\text { MSSSIM } \uparrow\end{array}$ & $\begin{array}{r}\text { Average } \\
\text { PSNR } \uparrow\end{array}$ & $\begin{array}{r}\text { Average } \\
\text { CQM } \uparrow\end{array}$ \\
\hline BB-SGD (ours) & $\mathbf{5 . 6 2 6 6}$ & $\mathbf{0 . 0 4 4 7}$ & $\mathbf{0 . 0 1 4 7}$ & $\mathbf{0 . 9 4 7 8}$ & $\mathbf{3 0 . 4 0 1 6}$ & $\mathbf{3 1 . 2 4 2 0}$ \\
SPMD [1] & 6.0985 & 0.0487 & 0.0154 & 0.9412 & 29.8439 & 30.6499 \\
LabGen-OF [2] & 6.1897 & 0.0566 & 0.0232 & 0.9412 & 29.8957 & 30.7006 \\
FSBE [6] & 6.6204 & 0.0605 & 0.0217 & 0.9373 & 29.3378 & 30.1777 \\
BEWIS [8] & 6.7094 & 0.0592 & 0.0266 & 0.9282 & 28.7728 & 29.6342 \\
Photomontage & 7.1950 & 0.0686 & 0.0257 & 0.9189 & 28.0113 & 28.8719 \\
[7] & & & & & & \\
SOBS [10] & 7.5183 & 0.0711 & 0.0242 & 0.9160 & 27.6533 & 28.5601 \\
Temporal Me- & 8.2761 & 0.0984 & 0.0546 & 0.9130 & 27.5364 & 28.4434 \\
dian Filter [20] & & & & & & \\
\hline
\end{tabular}

We provide a comparison of the proposed model with models which are fully unsupervised, i.e. which do not use a supervised segmentation model (such as LabGensemantic) and do not require a direct human supervision (such as MSCL). The proposed model, named BB-SGD (Background bootstrapping using stochastic gradient descent) gets a better average score than all referenced unsupervised models on all criteria as shown in Table 1. Table 2 lists AGE results per category of the SBMnet dataset. It shows that the proposed models gets better AGE results than all referenced unsupervised models on 4 categories : basic, clutter, background motion and short video, with a $15 \%$ accuracy improvement on the short video category compared to the best unsupervised model in this category, which illustrates the efficiency of the bootstrapping mechanism 
Table 2. evaluation results for the AGE criteria per category on the SBMnet 2016 dataset

\begin{tabular}{lrrrrrrrr}
\hline Method & Basic & $\begin{array}{r}\text { Intermittent } \\
\text { Motion }\end{array}$ & Clutter & Jitter & $\begin{array}{r}\text { Illumination } \\
\text { Changes }\end{array}$ & $\begin{array}{r}\text { Background } \\
\text { Motion }\end{array}$ & $\begin{array}{r}\text { Very } \\
\text { Long }\end{array}$ & $\begin{array}{r}\text { Very } \\
\text { Short }\end{array}$ \\
\hline BB-SGD (ours) & $\mathbf{3 . 7 8 8 1}$ & 4.8898 & $\mathbf{3 . 8 7 7 6}$ & 9.5374 & 4.5227 & $\mathbf{8 . 5 6 0 7}$ & 5.6494 & $\mathbf{4 . 1 8 7 2}$ \\
SPMD [1] & 3.8141 & $\mathbf{4 . 1 8 4 0}$ & 4.5998 & 9.8095 & $\mathbf{4 . 4 7 5 0}$ & 9.9115 & 6.0926 & 5.9017 \\
LabGen-OF [2] & 3.8421 & 4.6433 & 4.1821 & 9.2410 & 8.2200 & 10.0698 & 4.2856 & 5.0338 \\
FSBE [6] & 3.8960 & 5.3438 & 4.7660 & 10.3878 & 5.5089 & 10.5862 & 6.9832 & 5.4912 \\
BEWIS [8] & 4.0673 & 4.7798 & 10.6714 & 9.4156 & 5.9048 & 9.6776 & $\mathbf{3 . 9 6 5 2}$ & 5.1937 \\
Photomontage & 4.4856 & 7.1460 & 6.8195 & 10.1272 & 5.2668 & 12.0930 & 6.6446 & 4.9770 \\
[7] & & & & & & & & \\
SOBS [10] & 4.3598 & 6.2583 & 7.0590 & 10.0232 & 10.3591 & 10.7280 & 6.0638 & 5.2953 \\
Temporal Me- & 3.8269 & 6.8003 & 12.5316 & $\mathbf{9 . 0 8 9 2}$ & 12.2205 & 9.6479 & 6.9588 & 5.1336 \\
dian Filter [20] & & & & & & & & \\
\hline
\end{tabular}

introduced in the proposed model considering that for these sequences, the optical flow weights and global weights are not used and no frame is suppressed.

\subsection{Evaluation on SBI dataset}

The SBI dataset is composed of 14 image sequences. Ground truth backgrounds are available for all sequences. We use the Matlab tool available on the SBI website for fair comparison with other models, but do not report the CQM results considering that other sequences were evaluated with a Matlab tool which included a bug for the CQM computation, as indicated in the SBI website. We run the proposed model on the SBI dataset using the same hyperparameters as those used for the SBMnet dataset. The results of this evaluation are listed in Table 3 and show that the proposed model gets better results than all other unsupervised models for which an evaluation is available on the SBI website. We also evaluate the LabGen-OF model on this dataset using the public source code available for this model on Github and obtain the same conclusion.

Table 3. evaluation results per criteria on the SBI dataset. $\downarrow$ indicates lower score is better, $\uparrow$ indicates higher score is better.

\begin{tabular}{lrrrrr}
\hline Method & $\begin{array}{r}\text { Average } \\
\text { AGE } \downarrow\end{array}$ & $\begin{array}{r}\text { Average } \\
\text { pEPs } \downarrow\end{array}$ & $\begin{array}{r}\text { Average } \\
\text { pCEPS } \downarrow\end{array}$ & $\begin{array}{r}\text { Average } \\
\text { MSSSIM } \uparrow\end{array}$ & $\begin{array}{r}\text { Average } \\
\text { PSNR } \uparrow\end{array}$ \\
\hline BB-SGD (ours) & $\mathbf{2 . 4 6 4 4}$ & $\mathbf{0 . 0 0 8 3}$ & $\mathbf{0 . 0 0 5 8}$ & $\mathbf{0 . 9 8 9 6}$ & $\mathbf{3 7 . 6 2 2 7}$ \\
LabGen-OF [2] & 2.7191 & 0.0145 & 0.0106 & 0.9824 & 35.9758 \\
LabGen [3] & 2.9945 & 0.0139 & 0.0092 & 0.9764 & 35.2028 \\
BEWIS [8] & 3.8665 & 0.0242 & 0.0142 & 0.9675 & 32.0143 \\
Photomontage [7] & 5.8238 & 0.0469 & 0.0372 & 0.9334 & 31.8573 \\
SOBS [10] & 3.5023 & 0.0415 & 0.0222 & 0.9765 & 35.2723 \\
Temporal Median & 10.3744 & 0.1340 & 0.1055 & 0.8533 & 28.0044 \\
Filter[20] & & & & & \\
\hline
\end{tabular}

\subsection{Ablation study}

In order to check the contribution of the various weights described in this paper, we provide results obtained using truncated versions of the proposed model while keeping the hyperparameters fixed : Version 0 does not use any weight and does not remove motionless frames, and is then equivalent to temporal median filtering. Version 1 uses only the optical flow weights and does not remove motionless frames. Version 2 uses both optical flow weights and global weights and does not remove motionless frames. Version 3 uses bootsrap weights, global weights and optical flow weights, but does not remove motionless frames. The AGE scores obtained by these truncated models on the 18 videos of the SBMnet dataset for which a ground truth is available 
Table 4. AGE scores obtained using various truncated versions of the algorithm on 18 SBMnet sequences where a ground truth background is available

\begin{tabular}{|c|c|c|c|c|c|c|}
\hline Category & video & \multicolumn{4}{|c|}{$\begin{array}{l}\text { truncated model } \\
\text { version }\end{array}$} & $\begin{array}{r}\text { full } \\
\text { model }\end{array}$ \\
\hline \multicolumn{7}{|l|}{ background motion } \\
\hline & advertisementBoard & 1.61 & 1.62 & 1.60 & 1.34 & 1.71 \\
\hline & 511 & 3.42 & 3.44 & 3.43 & 3.44 & 3.43 \\
\hline & Blurred & 1.80 & 1.69 & 1.68 & 1.68 & 1.61 \\
\hline 年 & Foliage & 32.87 & 5.86 & 3.62 & 3.41 & 3.37 \\
\hline & Board & 21.37 & 6.78 & 7.84 & 7.37 & 7.39 \\
\hline & $\begin{array}{l}\text { People and Fo- } \\
\text { liage }\end{array}$ & 31.36 & 9.66 & 3.75 & 2.54 & 2.60 \\
\hline & boulevardJam & 21.37 & 15.89 & 19.5 & 11.0 & 2.03 \\
\hline 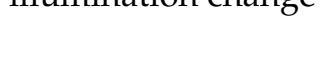 & CameraParameter & 11.49 & 22.19 & 2.16 & 2.81 & 2.95 \\
\hline & busStation & 5.31 & 5.40 & 5.47 & 5.67 & 5.32 \\
\hline & Candela_m1.10 & 4.93 & 5.09 & 5.18 & 5.21 & 2.81 \\
\hline & CaVignal & 12.57 & 12.61 & 13.58 & 14.04 & 2.05 \\
\hline & AVSS2007 & 10.98 & 10.32 & 10.25 & 10.01 & 8.73 \\
\hline \multicolumn{3}{|l|}{ jitter } & 2.00 & 1.93 & 1.74 & 1.84 \\
\hline & boulevard & 9.61 & 10.09 & 10.29 & 10.51 & 9.71 \\
\hline very long & BusStopMorning & 3.68 & 3.66 & 3.64 & 3.62 & 3.61 \\
\hline & Toscana & 8.79 & 8.80 & 8.79 & 3.30 & 3.30 \\
\hline & DynamicBackground & d 6.96 & 6.96 & 6.96 & 8.20 & 8.18 \\
\hline & CUHK_Square & 2.77 & 2.77 & 2.77 & 2.99 & 2.98 \\
\hline Average AGE by cat & ory & 8.06 & 7.53 & 4.94 & 4.51 & 3.75 \\
\hline
\end{tabular}


and using the evaluation tool available on the SBMnet website are provided in Table 4. They show that temporal median filtering (v0) gives the best results for five scenes, confirming that this is a good baseline. Introducing optical flow weights (v1) improves average AGE scores on scenes of the "clutter" category, but has no beneficial impact on other categories. Adding global weights (v2) has a positive impact on the "illumination change" category, which was expected, but also on the "clutter" category". Adding bootstrap weights has an impact on the "clutter" category, but also on the "short video" category. Finally, removing motionless frames, which leads to the full model, has a positive impact on the "intermittent motion" category, which was expected, but also on the scene "boulevardJam" of the "clutter" category, which also shows some intermittent motions.

\subsection{Computation time}

We have performed computation times measurements and tested the impact of reducing the number of optimization iterations, while keeping all other parameters frozen, excluding the learning rate. The results of these experiments are provided in Table 5. The total computation times necessary to reconstruct the 79 backgrounds from the associated video sequences of the SBMnet dataset is estimated by performing a sequential computation for all the videos, so that the computation times indicated in this table are the sum of the computation times of each of the 79 videos. If we divide the number of frames of the full dataset (73 355) with the total computation time of the proposed model, which is 1409 seconds, we get an average of 52 frames per second (fps). Table 5 shows however that the number of optimization iterations can be reduced from 3000 to 250 , increasing the average speed to $187 \mathrm{fps}$, without major impact on the overall accuracy of the algorithm. The computation times with such a low number of iterations are mainly associated with optical flow computations and JPEG images decoding.

Table 5. impact of reducing the number of iterations on average AGE score and computation time

\begin{tabular}{|c|c|c|c|c|c|}
\hline $\begin{array}{l}\text { Number of iterations } \\
\text { learning rate }\end{array}$ & $\begin{array}{l}100 \\
0.06\end{array}$ & $\begin{array}{l}250 \\
0.03\end{array}$ & $\begin{array}{l}500 \\
0.03\end{array}$ & $\begin{array}{l}1000 \\
0.03\end{array}$ & $\begin{array}{l}3000 \\
0.03\end{array}$ \\
\hline $\begin{array}{l}\text { Computation time for } 79 \text { videos of the } \\
\text { SBMnet dataset (seconds) }\end{array}$ & 337 & 391 & 482 & 666 & 1409 \\
\hline $\begin{array}{l}\text { Average AGE by category on } 18 \text { videos } \\
\text { of the SBMnet dataset listed in Table } 4\end{array}$ & 4.07 & 3.83 & 3.80 & 3.76 & 3.75 \\
\hline Average AGE on SBI dataset & 2.78 & 2.56 & 2.53 & 2.49 & 2.46 \\
\hline
\end{tabular}

Although the proposed model requires a GPU, these computation time measurements compare very favorably with the processing speeds reported by the authors of other models: The average computation speed of LabGen-OF is estimated to 5fps in [2]. The computation speed of SPMD is estimated in [1] to $1.6 \mathrm{fps}$ for $640 \times 480$ images and $22.8 \mathrm{fps}$ for 200x144 images using a Intel Core i7 2600@3.4Ghz CPU.

\subsection{Image samples}

Figure 2 shows some examples of background reconstruction for sequences of the SBMnet dataset, with the associated ground-truth when its is available and a comparison with the results obtained with LabGen-OF and SPMD.

\section{Conclusion}

We have presented a new algorithm for fixed background reconstruction using stochastic gradient descent which is simple, fast using a GPU, and more accurate than the state of the art. This shows that with modern hardware, stochastic gradient descent can be used efficiently for real-time applications and that the tools and frameworks which have been recently developed for deep learning and neural networks can also be 


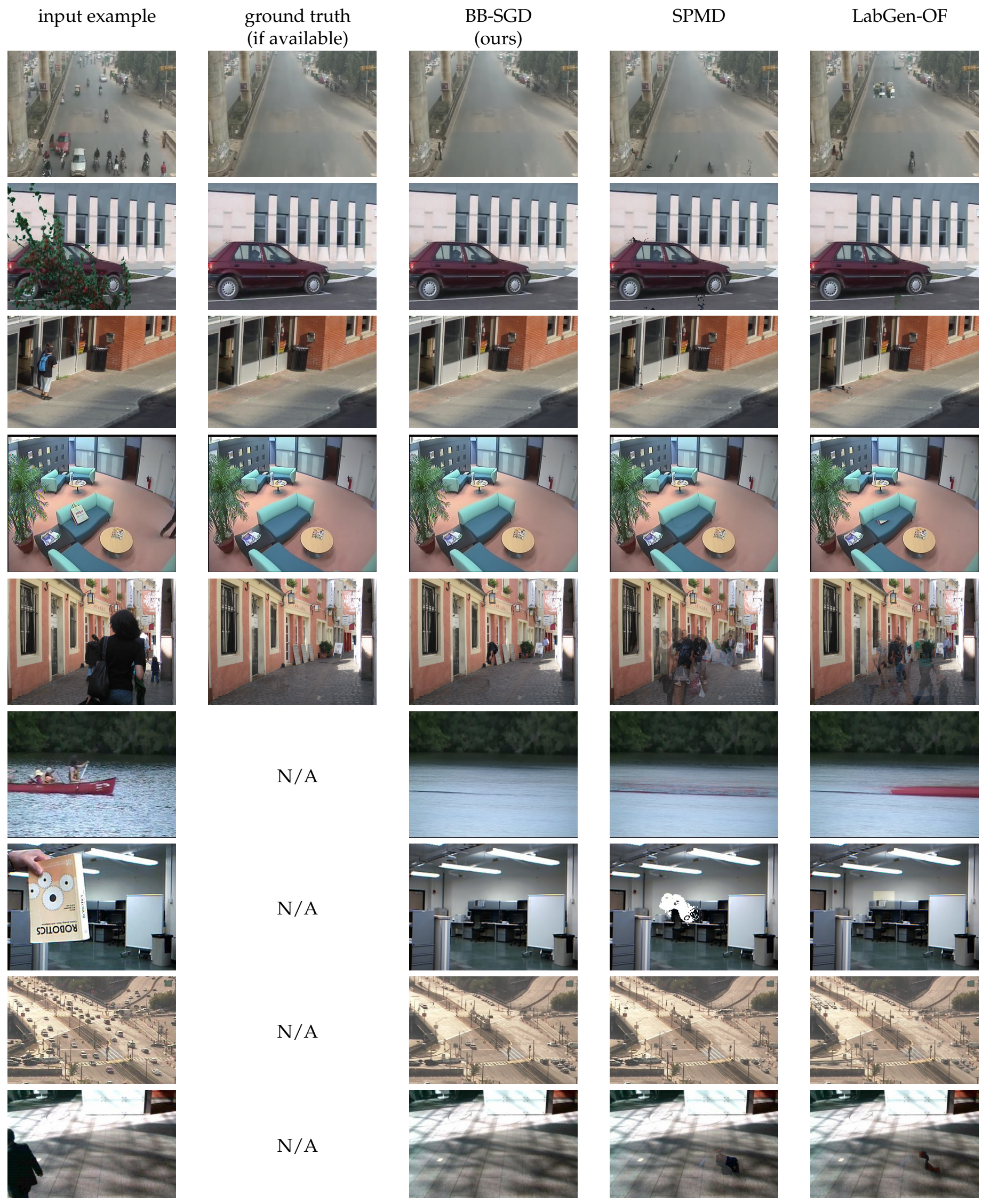

Figure 2. examples of background reconstruction using the proposed model and comparison with SPMD and LabGen-OF 
useful for other optimization problems with a proper design of the loss function. Further works include using the same approach to handle the task of dynamic background reconstruction and change detection.

Author Contributions: Conceptualization, B.S.; methodology, B.S.; software, B.S.; validation, B.S.; formal analysis, B.S.; investigation; writing—original draft preparation, B.S.; writing—review and editing, A.F.; visualization, B.S.; supervision, A.F.; project administration, A.F.; resources, A.F, funding acquisition, A.F.. All authors have read and agreed to the published version of the manuscript.

Funding: This research was funded by french National Research Agency (ANR)

Data Availability Statement: SBM.net dataset is available at the the following web address: http:/ / scenebackgroundmodeling.net/. The SBI dataset is available at the following web address: https://sbmi2015.na.icar.cnr.it/SBIdataset.html

Conflicts of Interest: The authors declare no conflict of interest.The funder had no role in the design of the study; in the collection, analyses, or interpretation of data; in the writing of the manuscript, or in the decision to publish the results.

\section{References}

1. Xu, Z.; Min, B.; Cheung, R.C. A robust background initialization algorithm with superpixel motion detection. Signal Processing: Image Communication 2019, 71, 1-12, [1805.06737]. doi:10.1016/j.image.2018.07.004.

2. Laugraud, B.; Van Droogenbroeck, M. Is a memoryless motion detection truly relevant for background generation with labgen? Lecture Notes in Computer Science (including subseries Lecture Notes in Artificial Intelligence and Lecture Notes in Bioinformatics) 2017, 10617 LNCS, 443-454. doi:10.1007/978-3-319-70353-4_38.

3. Laugraud, B.; Piérard, S.; Van Droogenbroeck, M. LaBGen: A method based on motion detection for generating the background of a scene. Pattern Recognition Letters 2017, 96, 12-21. doi:10.1016/j.patrec.2016.11.022.

4. Laugraud, B.; Piérard, S.; Van Droogenbroeck, M. Labgen-p-semantic: A first step for leveraging semantic segmentation in background generation. Journal of Imaging 2018, 4, 1-22. doi:10.3390/jimaging4070086.

5. Liu, W.; Cai, Y.; Zhang, M.; Li, H.; Gu, H. Scene background estimation based on temporal median filter with Gaussian filtering. Proceedings - International Conference on Pattern Recognition 2016, 0, 132-136. doi:10.1109/ICPR.2016.7899621.

6. Djerida, A.; Zhao, Z.; Zhao, J. Robust background generation based on an effective frames selection method and an efficient background estimation procedure (FSBE). Signal Processing: Image Communication 2019, 78, 21-31. doi:10.1016/j.image.2019.06.001.

7. Agarwala, A.; Dontcheva, M.; Agrawala, M.; Drucker, S.; Colburn, A.; Curless, B.; Salesin, D.; Cohen, M. Interactive digital photomontage. ACM SIGGRAPH 2004 Papers, SIGGRAPH 2004 2004, pp. 294-302. doi:10.1145/1186562.1015718.

8. De Gregorio, M.; Giordano, M. Background modeling by weightless neural networks. Lecture Notes in Computer Science (including subseries Lecture Notes in Artificial Intelligence and Lecture Notes in Bioinformatics) 2015, 9281, 493-501. doi:10.1007/978-3-319-232225_60.

9. Maddalena, L.; Petrosino, A. A self-organizing approach to background subtraction for visual surveillance applications. IEEE Transactions on Image Processing 2008, 17, 1168-1177. doi:10.1109/TIP.2008.924285.

10. Maddalena, L.; Petrosino, A. Extracting a background image by a multi-modal scene background model. Proceedings - International Conference on Pattern Recognition 2016, 0, 143-148. doi:10.1109/ICPR.2016.7899623.

11. Maddalena, L.; Petrosino, A. The SOBS algorithm: What are the limits? IEEE Computer Society Conference on Computer Vision and Pattern Recognition Workshops 2012, pp. 21-26. doi:10.1109/CVPRW.2012.6238922.

12. Halfaoui, I.; Bouzaraa, F.; Urfalioglu, O. CNN-based initial background estimation. Proceedings - International Conference on Pattern Recognition 2016, 0, 101-106. doi:10.1109/ICPR.2016.7899616.

13. Tao, Y.; Palasek, P.; Ling, Z.; Patras, I. Background modelling based on generative unet. 2017 14th IEEE International Conference on Advanced Video and Signal Based Surveillance, AVSS 2017 2017. doi:10.1109/AVSS.2017.8078483.

14. Javed, S.; Mahmood, A.; Bouwmans, T.; Jung, S.K. Background-Foreground Modeling Based on Spatiotemporal Sparse Subspace Clustering. IEEE Transactions on Image Processing 2017, 26, 5840-5854. doi:10.1109/TIP.2017.2746268.

15. Bouwmans, T.; Maddalena, L.; Petrosino, A. Scene background initialization: A taxonomy. Pattern Recognition Letters 2017, 96, 3-11. doi:10.1016/j.patrec.2016.12.024.

16. Bouwmans, T.; Javed, S.; Sultana, M.; Jung, S.K. Deep neural network concepts for background subtraction: A systematic review and comparative evaluation, 2019, [1811.05255]. doi:10.1016/j.neunet.2019.04.024.

17. Kroeger, T.; Timofte, R.; Dai, D.; Van Gool, L. Fast optical flow using dense inverse search. Lecture Notes in Computer Science (including subseries Lecture Notes in Artificial Intelligence and Lecture Notes in Bioinformatics) 2016, 9908 LNCS, 471-488, [1603.03590]. doi:10.1007/978-3-319-46493-0_29.

18. Jodoin, P.M.; Maddalena, L.; Petrosino, A.; Wang, Y. Extensive Benchmark and Survey of Modeling Methods for Scene Background Initialization. IEEE Transactions on Image Processing 2017, 26, 5244-5256. doi:10.1109/TIP.2017.2728181. 
19. Maddalena, L.; Petrosino, A. Towards benchmarking scene background initialization. Lecture Notes in Computer Science (including subseries Lecture Notes in Artificial Intelligence and Lecture Notes in Bioinformatics) 2015, 9281, 469-476, [1506.04051]. doi:10.1007/978-3-319-23222-5_57.

20. Piccardi, M. Background subtraction techniques: A review. Conference Proceedings - IEEE International Conference on Systems, Man and Cybernetics 2004, 4, 3099-3104. doi:10.1109/ICSMC.2004.1400815. 